\title{
TRATAMENTO DE SEMENTES DE FEIJOEIRO-COMUM COM FUNGICIDA, INSETICIDA E PROMOTORES DE CRESCIMENTO
}

\author{
Fernanda Silva MESQUITA $^{1}$ \\ Luciana Ribeiro COIADO ${ }^{2}$
}

Aurivan Soares de FREITAS ${ }^{3}$

Clayton Rabelo REIS ${ }^{4}$

Eliana ALCANTRA ${ }^{5}$

Ramiro Machado REZENDE ${ }^{6}$

\begin{abstract}
${ }^{1}$ Acadêmica do curso de graduação em Agronomia da Universidade Vale do Rio Verde - UninCor. nanda.smesquita@gmail.com

${ }^{2}$ Acadêmica do curso de graduação em Agronomia da Universidade Vale do Rio Verde - UninCor. luciana@terranova.agr.br

${ }^{3}$ Doutor em Fitopatologia. Professor da Universidade Vale do Rio Verde - UninCor. aurivan.soares@ hotmail.com

${ }^{4}$ Academico do curso de graduação em Agronomia da Universidade Federal de Lavras - UFLA. claytonrabeloreis@hotmail.com

${ }^{5}$ Doutora em Entomologia. Professora da Universidade Vale do Rio Verde - UninCor. lialcantra@ yahoo.com.br

${ }^{6}$ Doutor em Agronomia/Fitotecnia. Coordenador e professor do curso de Agronomia da Universidade Vale Rio Verde UninCor. coord.agronomia@unincor.edu.br
\end{abstract}

Recebido em: 06/07/2016 - Aprovado em: 30/11/2017 - Disponibilizado em: 30/12/2017

\section{RESUMO:}

$\mathrm{O}$ tratamento de sementes confere à planta melhores condições defesas, o que possibilita maior potencial para o desenvolvimento inicial da cultura. O objetivo deste estudo foi avaliar o efeito do tratamento de sementes na emergência, produtividade e na qualidade de sementes de feijão. O experimento foi realizado em delineamento experimental em blocos casualizados com 4 tratamentos e 5 repetições. Sementes da cultivar BRS Estilo foram tratadas com Initiate Soy®, Fludioxonil + Metaxil-M (Maxim XL®) e Thiamethoxam (Cruiser®), produtos com atividade na promoção de crescimento, fungicida e inseticida, respectivamente. Após tratadas, as sementes foram plantadas em uma área de $600 \mathrm{~m}^{2}$ com espaçamento de $0,5 \mathrm{~m}$ entre linhas e 13 sementes por metro. Avaliou-se a emergência aos 15 dias após o plantio, a produtividade e a qualidade das sementes. Os produtos que apresentaram melhores resultados para a emergência aos 15 dias após a semeadura foram Cruiser ${ }^{\circledR}+$ Maxim XL® e Cruiser® + Maxim XL® + Initiate Soy®. Apesar desses resultados, o tratamento de sementes não influenciou a qualidade das sementes e a produtividade do feijoeiro.

Palavras-chave: Phaseolus vulgaris L..Tecnologia de produção. Produtividade. Emergência.

\section{TREATMENT OF COMMON BEAN SEEDS WITH FUNGICIDE, INSECTICIDE AND GROWTH PROMOTERS}

\begin{abstract}
:
Seed treatment gives to the plant best conditions of defense, allowing greater potential for the initial development of the culture. The objective of this study was to evaluate the effect of seed treatment in emergency, productivity and quality of bean seeds. The experiment was conducted in a randomized block design with 4 treatments and 5 repetitions. The seeds designated BRS Estilo were treated with Initiate Soy®, Fludioxonil + metaxil-M (Maxim XL®) e Thiamethoxam (Cuiser ${ }^{\circledR}$ ), products with activity in promoting growth, fungicide and insecticide respectively. After treatment, the seeds were sown in an area of $600 \mathrm{~m}^{2}$ with spacing of $0.5 \mathrm{~m}$ between rows and 13 seed per meter. We evaluated 15 days after planting, the emergency productivity and quality of seeds. The products that showed better results for the emergency to 15 days after sowing were Cruiser® + Maxim XL® e Cruiser® + Maxim XL® + Initiate Soy®. Although these results, the seed treatment did not affect seed quality and productivity of bean.
\end{abstract}

Keywords: PhaseolusvulgarisL.. Productiontechnology. Productivity. Emergency. 


\section{INTRODUÇÃO}

O feijoeiro (Phaseolus vulgaris L.) é uma planta de grande expressividade, extrapolando os aspectos econômicos por sua relevância quanto ao fator de importância alimentar sendo uma grande fonte de proteínas, vitaminas e minerais. Seu cultivo está difundido em todo o Brasil, fato que torna uma planta de grande importância para famílias de baixa renda. Nos últimos anos, os produtores vêm adotando novas tecnologias com objetivo de aumentar à produção e consequentemente a qualidade dos grãos (BARBOSA, 2012).

Dentre as condições necessárias para o cultivo na face inicial, a semente exerce um papel fundamental do estande no campo, sendo que sementes infectadas por patógenos apresentam viabilidade e vigor inferiores. Além de ser meio de transporte para patógenos, podem ser também alimentos para pragas de solo, que podem se alimentar de sementes e raízes e da parte área das plantas (PESKE et al., 2009).

A aplicação de produtos no tratamento de sementes, com a finalidade de aumentar a produção tem se tornado uma prática agrícola cada vez mais constante. Entre os produtos, destacam-se fungicidas, inseticidas, inoculantes, antibióticos, hormônios e aminoácidos (BERNADES et al., 2010).

A utilização de inseticidas para o tratamento de sementes é uma maneira de reduzir a perda decorrente das ações de insetos que danificam as plantas jovens. $\mathrm{O}$ uso do inseticida a base de Thiomethoxam, vem mostrando um poder de atuação fisiológica nas plantas caracterizando uma ação positiva no crescimento e desenvolvimento das plantas (BARROS et al., 2001; CASTRO et al., 2008).

Segundo Moraes et al. (2003) vários fungicidas têm sido eficientes no controle de fungos presentes nas sementes. De acordo com esses autores, o fungicida a base de Fludioxonil + Metaxil-M reduziu os danos causados pela associação do fungo Fusarium moniliforme em sementes de milho.

Além disso, novas tecnologias, como o uso de controladores hormonais têm merecido cada vez mais atenção na agricultura à medida que as técnicas de cultivo evoluem, principalmente, em culturas de alto valor. Esses produtos diferenciam-se como biorreguladores, bioestimulantes e bioativadores (ALMEIDA et al., 2011). Os estudos indicam que os controladores hormonais aceleraram o desenvolvimento das plantas, resultando em acréscimo na produção (LANA et al., 2009).

Diante dessas informações, objetivouse com este trabalho avaliar a emergência, a produtividade e a qualidade de sementes de feijão por meio do tratamento com Thiomethoxam Cruiser®, Fludioxonil + Metaxil-MMaxim XL® e o promotor de crescimento Initiate Soy® 


\section{REVISÃO DE LITERATURA}

No Brasil, o feijoeiro-comum é cultivado por agricultores de diversos perfis, em diferentes escalas, regiões e sistemas de produção. O Brasil é o maior produtor e consumidor de feijão do mundo. A produção brasileira na safra 2015/2016 chegou ao patamar de 3.054.200 toneladas, com produtividade média de 1023,5 kg/ha (CONAB, 2016).

Dependendo da região pode se encontrar até três safras de feijão durante o ano. A safra das "águas" ou a $1^{\text {a }}$ safra com plantio nos meses de agosto a novembro e colheita de novembro a fevereiro, safra da "seca" ou $2^{\mathrm{a}}$ safra com plantio de dezembro a março e colheita de março a junho e por último a safra de inverno que também pode ser conhecida por safra de $3^{\text {a }}$ época ou safra irrigada com plantio de abril a julho e colheita de julho a outubro (SILVA, 2005).

As doenças estão dentre os fatores limitantes da produção do feijoeiro, levando a perdas no rendimento e na qualidade do produto. Os patógenos transmitidos pelas sementes são de expressiva importância. Assim, o tratamento de sementes com fungicidas se tornou a maneira mais eficaz e econômica de controle (KINTSCHEV et al., 2014). Além de eliminar ou reduzir o inóculo inicial das doenças, pode proteger a plântula dos patógenos existentes no solo (BARROS, 2001).
Os danos causados por insetos também alteram o sistema reprodutivo e vegetativo da planta, reduzindo a produção e a qualidade das sementes. De forma indireta, são responsáveis pela transmissão de vírus, que ocasionam perdas de até $100 \%$ da produção (BARBOSA et al., 2002).

O tratamento de sementes com inseticidas também é um método muito utilizado e reduz possíveis perdas das ações de vários insetos-pragas, que causam enormes danos às áreas subterrâneas do feijoeiro, desde a germinação, até as plantas jovens (BARROS et al., 2001).

Os tratamentos de sementes com agrotóxicos têm como vantagens, diminuição das falhas da germinação, melhor aproveitamento de equipamentos utilizados na semeadura, além dos custos adicionais mais econômicos. Alguns produtos têm efeito na nutrição da planta, aumentando a absorção dos nutrientes existentes no solo.

Com a grande demanda do feijoeiro, tecnologias visando o aumento da produção, enriquecidos com aminoácidos e minerais, utilizados via foliar ou via tratamento de sementes, estão sendo usados para estimular o desenvolvimento e crescimento de plantas e raízes de culturas como o feijoeiro. Com a possibilidade de uma melhor absorção de água e nutrientes pelas plantas, ou até com tolerância ao déficit hídrico, espera-se uma melhor produtividade para o feijoeiro (LIMA et al., 2011). 
Ainda pouco explorado, por ser recém-desenvolvido, ouso de promotores de crescimento tem apresentado resultados promissores. De acordo com Weber (2012) tem-se observado maior produtividade $\mathrm{e}$ ganho de massa, em função do fornecimento de nutrientes essenciais para a planta, tanto na etapa germinativa como na reprodutiva. Espera-se, um melhor desenvolvimento radicular das plantas e um rápido estabelecimento da cultura.

\section{MATERIAL E MÉTODOS}

$\mathrm{O}$ experimento foi conduzido na Fazenda Limeira, município de Monsenhor Paulo - MG, entre os meses de janeiro a abril de 2016. O solo da área experimental é classificado como Cambissolo Háplico de textura argilosa.

Antes do plantio havia uma lavoura de café cultivar Mundo Novo IAC 376-4 com idade de cinquenta anos, que foi retirada no dia 10 de outubro de 2015. Os resultados da análise química da camada de $0-20 \mathrm{~cm}$ apresentaram: $\mathrm{pH}\left(\mathrm{CaCl}_{2}\right)=4,8 ;$ M.O $=1,7$ $\mathrm{mg} \mathrm{dm}^{-3} ; \mathrm{P}=7,0 \mathrm{mg} \mathrm{dm}^{-3} ; \mathrm{H}+\mathrm{Al}=3,2 \mathrm{cmol}_{\mathrm{c}}$ $\mathrm{dm}^{-3} ; \mathrm{K}=164 \mathrm{mg} \mathrm{dm}^{-3} ; \mathrm{Ca}=2,0 \mathrm{cmol}_{\mathrm{c}} \mathrm{dm}^{-3}$; $\mathrm{Mg}=0,4 \mathrm{cmol}_{\mathrm{c}} \mathrm{dm}^{-3} \mathrm{e} ; \mathrm{V} \%=46,7 . \mathrm{A}$ calagem foi realizada 90 dias antes do plantio aplicando-se 3,0 t ha ${ }^{-1}$ de calcário dolomítico, conforme análise de solo. O preparo do solo consistiu de uma aração seguida de uma gradagem e duas passagens da grade niveladora. A adubação de plantio foi feita com a aplicação de $375 \mathrm{Kg} \mathrm{ha}^{-1}$ de NPK (0824-12) e a cobertura foi realizada 30 dias após o plantio aplicando-se $100 \mathrm{Kg} \mathrm{ha}^{-1}$ de NPK (30-00-20) seguindo a Quinta Aproximação. Foi semeada a cultivar BRS Estilo, de ciclo médio, porte ereto, hábito de crescimento indeterminado tipo II e resistente ao mosaico comum.

O delineamento experimental foi $\mathrm{em}$ blocos casualizados, com quatro tratamentos e cinco repetições. Cada parcela continha seis linhas de $5 \mathrm{~m}$, espaçadas por $0,5 \mathrm{~m}$ entre linhas, na qual foram semeadas 13 sementes por metro. A área útil foi constituída pelas quatro linhas centrais desprezando-se $0,5 \mathrm{~m}$ de cada lado, totalizando $6 \mathrm{~m}^{2}$ de área útil.

Foram avaliados quatro tratamentos de sementes correspondentes da seguinte forma, para cada tratamento continha $700 \mathrm{~g}$ de sementes $\mathrm{e}$ as dosagens foram: $\mathrm{T} 1=$ testemunha sem adição de produtos; $\mathrm{T} 2=$ Promotor de crescimento Initiate Soy® 2,5 $\mathrm{mL} ; \mathrm{T} 3=$ Cruiser® 2,0 mL + Maxim XL® 3,0 mL; T4 = Cruiser ${ }^{\circledR}$ 2,0 $\mathrm{mL}+$ Maxim XL® 3,0 mL + Initiate Soy® 2,5 mL. As sementes foram separadas e tratadas em saquinhos plásticos antes do plantio para homogeneizar os produtos, e a semeadura foi realizada de forma manual.

As plantas daninhas de folhas estreitas foram controladas mediante aplicação $200 \mathrm{~mL}$ $\mathrm{ha}^{-1}$ de herbicida (FLUAZIFOPE-PBUTÍLICO®). Já as de folhas largas foram controladas com capina manual e roçadora na 
entre linhas e carreadores. Não foram realizadas aplicações de agrotóxicos o desenvolvimento da cultura há não ser via tratamento de sementes.

As variáveis analisadas foram: emergência aos 15 dias após o plantio, a produtividade $\left(\mathrm{kg} \mathrm{ha}^{-1}\right) \mathrm{e}$, a qualidade das sementes. A emergência foi analisada contando-se o número de plantas da área útil de cada parcela. Para avaliar a produtividade, as sementes foram secas até $13 \%$ de umidade e pesadas em balança de precisão. A qualidade das sementes foi analisada, pesando-se 100 grãos coletados aleatoriamente de cada parcela.

As variáveis foram submetidas à análise de variância (ANOVA). As variáveis significativas no teste $\mathrm{F}$ foram comparadas, por meio do teste de Scott-Knott, a 5\% de probabilidade. As análises foram realizadas utilizando-se o programa Sisvar (FERREIRA, 2011).

\section{RESULTADOS E DISCUSSÃO}

A variável emergência foi influenciada pelos tratamentos de sementes. Os tratamentos que apresentaram os melhores resultados para a emergência aos 15 dias após a semeadura foram Cruiser ${ }^{\circledR}+$ Maxim XL® e
Cruiser ${ }^{\circledR}+$ Maxim XL® + Initiate Soy® como se pode observar na (Gráfico1).

Os diferentes tipos de tratamento de sementes não influenciaram a produtividade $\mathrm{e}$ o peso de 100 grãos. A produtividade foi $1849,9,1766,6,2316,6$ e 2100,0 e o peso de 100 grãos foi $20,2,20,8,20,6$ e 20,8 na testemunha e nos tratamentos de sementes com Initiate Soy®, Cruiser ${ }^{\circledR}$ (Thiametoxan) + Maxim XL® (Fludioxanil Metalaxil-M) e Cruiser® (Thiametoxan) + Maxim XL® (Fludioxanil Metalaxil-M) + Initiate Soy®, respectivamente (Tabela 1 ).

Gráfico 1 - Emergência de sementes de feijão em razão do tratamento com fungicida, inseticida e promotores de crescimento. Médias seguidas de letra, não diferem estatisticamente entre si pelo teste de Scott-Knott 5\% de probabilidade, CV\%: 9,79.

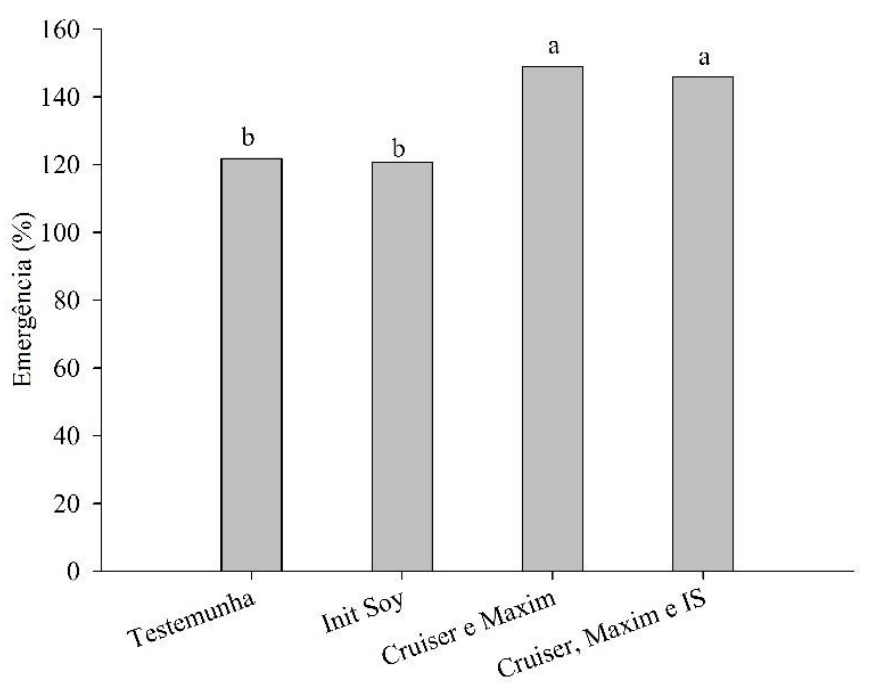


Tabela 1 - Produtividade e peso dos grãos em razão do tratamento com fungicida, inseticidas e promotores de crescimento, não significativo ao nível de $5 \%$ de probabilidade pelo teste $\mathrm{F}$.

\begin{tabular}{lcc} 
Tratamentos & Produtividade Kg ha & Peso 100 grãos g $^{-1}$ \\
\hline 1- Testemunha & 1849,9 & 20,2 \\
2- Initiate Soy & 1766,6 & 20,8 \\
3- Cruiser + Maxim XL & 2316,6 & 20,6 \\
4- Cruiser + Maxim XL + Initiate Soy & 2100 & 20,8 \\
\hline Média & 2008,32 & 20,6 \\
\hline CV $(\%)$ & 16,72 & 4,91 \\
\hline
\end{tabular}

No presente trabalho, o uso do inseticida (Cruiser®) (Thiamethoxam juntamente com o fungicida (Maxim $\left.{ }^{\circledR}\right)$ Fludioxanil Metalaxil-M), apresentou um efeito positivo no tratamento de sementes de feijoeiro. De forma semelhante, Barros et al. (2001) evidenciaram que o tratamento de sementes de feijão como Thiamethoxam, Fludioxonil + Metalaxil-M promoveu os mais elevados níveis de controle de fungos, bem como o controle da mosca branca. Apesar do trabalho atual não trazer dados sobre controle de doenças e pragas, ficou visível seu efeito nos resultados da emergência quando se usou inseticida mais fungicida.

Houve um diferencial, em relação à emergência, quando comparado a testemunha,ou só do promotor de crescimento Initiate Soy® e o inseticida Thiamethoxam, identificou-se um número maior de plantas emergidas, visto que no experimento realizado por Almeida et al. (2011), com semente de arroz, o uso do inseticida apresentou um desempenho superior, a germinação inicial de diferentes cultivares de arroz que estavam abaixo de $90 \%$ e com o tratamento, chegou até $96 \%$. Diferentemente do trabalho realizado por Binsfeld et al. (2014), o Thiamethoxam apresentou efeito negativo em sementes de soja, tanto na germinação, quanto no desenvolvimento das plântulas.

O promotor de crescimento Initiate Soy® não atingiu resultados expressivos, apresentando resultados estatisticamente iguais a testemunha). Weber (2012), utilizou quatro diferentes enraizadores, mas não verificou resultados significativos, mas uma boa evolução da cultura.

No presente trabalho houve diferença significativa apenas para a emergência, não apresentando diferença expressiva para as demais variáveis. Como sugere Weber (2012), é necessário realizar novos testes, com diferentes produtos e combinações, para um melhor desempenho agronômico da cultura do 
feijoeiro com tratamentos de sementes, como já foi obtido em outras culturas.

\section{CONCLUSÃO}

O tratamento de sementes de feijão com o inseticida Thiomethoxam + fungicida Fludioxanil Metalaxil-M e Thiametoxam + Fludioxanil Metalaxil-M + promotor de crescimento Initiate Soy® melhoram a

\section{REFERÊNCIAS}

ALMEIDA, A. S et al. Bioativador no desempenho fisiológico de sementes de arroz. Revista Brasileira de Sementes. v. 33, n. 3, 2011.

BARBOSA, F. R.; GONZAGA, A. C. O. Informações técnicas para o cultivo do feijoeiro-comum na Região CentralBrasileira: 2012-2014. Embrapa Arroz e Feijão-Documentos (INFOTECA-E), 2012.

BARBOSA, F. R et al. Efeito do controle químico da mosca-branca na incidência do vírus-do-mosaico-dourado e na produtividade do feijoeiro. Pesquisa Agropecuária Brasileira. v. 37, n. 6, 2002.

BARROS, R. G.; YOKOYAMA, M.; COSTA, J. L. S. Compatibilidade do inseticida thiamethoxan com fungicidas utilizados no tratamento de sementes de feijoeiro. Pesquisa Agropecuária Tropical. v. 31, n. 2, p. 153-157, 2001.

BERNARDES, Tatiely Gomes; DA SILVEIRA, Pedro Marques; MESQUITA, Marcos Antônio Machado. Regulador de crescimento e Trichoderma harzianum aplicados em sementes de feijoeiro cultivado em sucessão a culturas de cobertura. Pesquisa Agropecuária Tropical, Goiânia, v. 40, n. 4, p. 439-446, 2010.

BINSFELD, J. A et al. Uso de bioativador, bioestimulante e complexo de nutrientes em emergência das plântulas de feijão em relação a testemunha e ao tratamento que se utilizou somente o Initiate Soy®.

O tratamento de sementes com Initiate Soy®, Fludioxanil + Metaxil-M (Maxim XL®) e Thiamethoxam (Cruiser®) não influenciou a qualidade das sementes e a produtividade do feijoeiro em relação à testemunha.

sementes de soja. Pesquisa Agropecuária Tropical. v. 1, n. 1, 2014.

CASTRO, G. S. A et al. Tratamento de sementes de soja com inseticidas e um bioestimulante. Pesquisa Agropecuária Brasileira. v. 43, n. 10, 2008.

\section{CONAB, Companhia Nacional de} Abastecimento, <http://www.conab.gov.br/OlalaCMS/uploads /arquivos/16_06_09_16_49_15_boletim_grao s_junho_2016_-_final.pdf. >. Acesso em junho 2016.

FERREIRA, D. F. Sisvar: a computer statistical analysis system. Ciência e Agrotecnologia. v. 35, n. 6, 2011.

KINTSCHEV, M. R et al. Compatibilidade entre a inoculação de rizóbios e fungicidas aplicados em sementes de feijoeiro-comum, 2014.

LANA, A. M. Q et al. Aplicação de reguladores de crescimento na cultura do feijoeiro. Bioscience Journal. v. 25, n. 1, 2009.

LIMA, D. A. P. et al. Promotores de crescimento na produtividade do feijoeiro comum. In: Embrapa Arroz e Feijão-Artigo em anais de congresso (ALICE). In: CONGRESSO NACIONAL DE PESQUISA DE FEIJÃO, 10., 2011, Goiânia. Anais... Goiânia: Embrapa Arroz e Feijão, 2011. 
MORAES, M. H. D et al. Controle químico de Fusarium moniliforme em sementes de milho: metodologia de avaliação e efeitos sobre a qualidade fisiológica. Fitopatologia Brasileira. v. 28, n. 6, 2003.

PESKE, F. B.; BAUDET, L.; PESKE, E. Produtividade de plantas de soja provenientes de sementes tratadas com fósforo. Revista Brasileira de Sementes. v. 31, n. 1, 2009.

SILVA, C. C.; PELOSO, M. J. D. Informações técnicas para o cultivo do feijoeiro comum na Região Central-brasileira 2005-2007. 16. Reunião da Comissão Técnica Central-brasileira de Feijão Goiânia, GO (Brasil). 17-21, 2005.

WEBER, M. H. B. Eficiência do uso de enraizadores na cultura do feijão, 2012. 